\section{Adapt current tools for handling big data}

To speed up discoveries of disease biomarkers and treatments, we must work out a cheaper and faster way to process, store and use the huge medical data sets that are rapidly becoming available (see, for example, Nature 506, 144-145; 2014).

By 2015, it is likely that a typical hospital will create 665 terabytes of data a year (for comparison, the web archive of the US Library of Congress contains less than 500 terabytes). This information can be used to study and analyse treatments - for example, for tuberculosis and stroke - and to reduce health-care costs.

To handle such big data effectively (see also Nature 498, 255-260; 2013), we need to adapt classical information-processing tools. One computational challenge is how to manage the huge volume of detailed material as it becomes available, without sacrificing information. Another is that the data mostly represent physiological processes, the characteristics of which change over time.

Current methods are also inadequate for analysing collective information from different sensors, such as multidimensional descriptions from electroencephalography or magnetic resonance imaging of interactions between brain regions.

Ervin Sejdić University of Pittsburgh, Pennsylvania, USA. esejdic@ieee.org

\section{Portugal's research funding is secure}

As president of the Portuguese Foundation for Science and Technology (FCT), I disagree with André Levy's overly pessimistic view of prospects for young scientists in the country (Nature 506, 159; 2014).

The cuts in $\mathrm{PhD}$ studentships and postdoctoral fellowships that Levy mentions have been partially offset by FCT-funded $\mathrm{PhD}$ programmes and a funding boost for the latest round of fellowships. Plenty of opportunities will arise for recruitment and retention of young scientists through the steady funding of research projects and centres by the FCT, and from increased international funding through programmes such as the European Union's Horizon 2020 scheme.

In the past two years, the FCT has launched initiatives to strengthen Portugal's research base: $\mathrm{PhD}$ programmes and career-development schemes; differentiated research grants; a rigorous evaluation of centres of excellence; and top-up incentives for centres that bring in European or non-public funding. We are also building a national road map for research infrastructure.

The exponential growth of research and development in Portugal in the past $20-30$ years is set to continue as the challenges of quality and international competitiveness are addressed. Miguel Seabra FCT, Lisbon, Portugal.

presidencia@fct.pt

\section{Forests are more than sticks of carbon}

Valentin Bellassen and Sebastiaan Luyssaert suggest that forest managers should improve both carbon stocks and timber harvests to mitigate climate change (Nature 506, 153-155; 2014). But forests are more than just sticks of carbon.

Maximizing harvests may be appropriate in heavily managed plantations. But in the remaining $96 \%$ of the world's forests, it could conflict with other forest uses and ecosystem services, as well as with biodiversity.

Some 1.6 billion people depend on forests to live. So optimizing wood production and carbon stocks at the expense of traditional uses and access rights could backfire. These people should not become carbon refugees.
The authors recommend "protecting trees from animals". In the African and Asian tropics particularly, such a move would have to consider the needs of elephants, rhinoceroses and ungulates, for example, to avoid undermining conservation efforts and the crucial ecosystem functions that these animals provide, such as seed and nutrient dispersal.

There is also a risk that simplifying forests to optimize two ecosystem services would reduce forest resilience to pest outbreaks and droughts, and to climatic and environmental changes (R. F. Noss Conserv. Biol. 15, 578-590; 2001).

Promoting areas of multi-use forest that have various functions would provide environmental and social benefits, while retaining more carbon than smaller, single-use areas.

Aerin L. Jacob, Sarah Jane Wilson McGill University, Montreal, Canada. aerin.jacob@mail.mcgill.ca Simon L. Lewis University College London, UK.

\section{Focus on questions, not hypotheses}

I contend that the insistence of the US National Institutes of Health (NIH) on hypothesisdriven projects in grant proposals could be a factor contributing to irreproducible research reports (see F. S. Collins and L. A. Tabak Nature 505, 612-613; 2014).

Isaac Newton argued that "hypotheses... have no place in experimental philosophy”, a view echoed by mathematician Roger Cotes: "Those who assume hypotheses as first principles of their speculations ... may indeed form an ingenious romance, but a romance it will still be" (in I. B. Cohen Introduction to Newton's Principia; iUniverse, 1999).

Such criticisms recognize the risk that scientists may filter data through their hypotheses, discounting results that do not validate the hypothesis as evidence that the experiment did not work - rather than as evidence that the hypothesis is false.

The NIH's funding criteria should instead ensure that a pertinent research question is being asked, and that the applicant has the means to answer it (see D. J. Glass Experimental Design for Biologists; Cold Spring Harbor Laboratory Press, 2006).

David J. Glass Novartis Institutes for Biomedical Research, Cambridge, Massachusetts, USA. david.glass@novartis.com

\section{Update forensics for deaths in Japan}

Autopsies in Japan are performed on only $1.6 \%$ of all deaths, the lowest rate among developed countries. Cause of death is almost always determined by simple visual inspection, as it was 100 years ago. Japanese forensic medicine needs to catch up to avoid murders being overlooked.

In 2011, the National Police Agency announced that 43 murders had been missed since 1998, with many found to have been disguised as suicides or natural deaths.

The agency is now trying to carry out more autopsies, but examinations are often cursory because of budget restrictions. To tackle the problem, rigorous autopsies need to be performed routinely.

In 2012, two laws relating to death investigations were passed, and the government established the Committee for the Promotion of Cause of Death Investigation, which published interim reports in May 2013. No governmental agency will take responsibility for the budget, however, so little progress has been made.

Exposing the possible involvement of crime in unnatural deaths continues to be as hard as it ever was in Japan.

Hiroshi Ikegaya Kyoto

Prefectural University of

Medicine, Kyoto, Japan. ikegaya@koto.kpu-m.ac.jp 